\title{
Comparison Between the Transperitoneal and Retroperitoneal Approach Methods for Severe Retroperitoneal Abscess
}

\author{
Kyungtaek Kim, Sungtaek Jung *, Keunmyung Park \\ Department of Surgery, Inha university hospital, Incheon, Korea
}

\section{Article history:}

Recevied: August 18, 2020

Revised: October 12, 2020

Accepted: October 27, 2020

\section{${ }^{*}$ Corresponding Author:}

Sungtaek Jung

Department of Surgery, Inha university hospital, 27, Inhang-ro, Jung-gu, Incheon, Korea

E-mail: habana95@hanmail.net

\section{ORCID}

Kyungtaek Kim

https://orcid.org/0000-0003-2740-6644

Sungtaek Jung

https://orcid.org/0000-0001-7035-8209

Keunmyung Park

https://orcid.org/0000-0002-9581-0019

\begin{abstract}
Purpose: Retroperitoneal (RP) abscess is a rare condition with poor prognosis, unclear etiology, characteristics, and treatment. This study compared transperitoneal and RP approach methods in the treatment of RP abscess.

Methods: There were 45 patients with RP abscess diagnosed at Inha University Hospital from January 2014 to August 2018, of which 22 patients with 2 RP zones and systemic inflammatory response syndrome were included. Characteristics, etiology, laboratory and radiological findings, surgical technique, complications, and total number of hospital days were examined. Patients were assigned to either the transperitoneal (TP) or RP approach group.

Results: There were 22 patients with RP abscess who were treated with surgical drainage using either the TP $(n=13)$ or RP ( $n=9)$ approach. There was no difference in characteristics between the 2 groups. The number of reinterventions in the RP group $(n=6)$ was more than in the TP group ( $n=4$; $p=0.02)$. Mortality in the TP group $(n=5)$ was higher than in the RP group $(n=1 ; p<0.01)$. The total mean number of hospital days in the RP group (mean: 76, range: 29-180 days) was more than in the TP group (mean: 58 , range: $18-280$ days, $p=0.03$ ).
\end{abstract}

Conclusion: RP abscess requires rapid drainage using the TP or RP approach. Reintervention events and number of hospital days in the RP group was greater than the TP group; however, mortality in the RP group was lower than in the TP group.

Keywords: abscess, drainage, operative surgical procedure, retroperitoneal space

\section{Introduction}

Retroperitoneal (RP) abscess is a rare condition where there is an insidious onset of symptoms and difficulty in diagnosis which delays treatment resulting in a poor prognosis. Delayed diagnosis or inadequate treatment of infection in the retroperitoneum and the organs contained within, such as the kidney, can lead to abscess formation, particularly in immunocompromised patients. Abscess formation results in a greater risk of morbidity and, if not drained promptly, increased mortality. In well-resourced countries, these risks have been reduced by ready access to computed tomography (CT), which can help to rapidly diagnose abscess formation and guide drainage [1]. RP abscesses can occur due to the spread of infection from adjacent organs. Known causes of RP abscesses include renal infarctions, osteomyelitis, colon carcinoma, diverticulitis, Crohn's disease, pancreatitis, and appendicitis.

There are 3 ways to treat an abscess: conservative, percutaneous drainage and surgical treatment. If the size of the abscess is less than $3 \mathrm{~cm}$, the conservative approach with antibiotic therapy is effective. Percutaneous drainage, due to its high-resolution rate, has been reported in recent years to be the best treatment for an abscess, however, percutaneous drainage is not always possible [2]. Treatment of an abscess using surgical drainage involves 2 different methods which have been considered with advantages and disadvantages. The transperitoneal (TP) approach is the most direct however, the viscera must be retracted. The RP approach can be 
implemented with a longer dissection to avoid viscera matter [3]. A more effective surgical method for treating RP abscess has not yet been established.

The objective of this study was to compare the TP and RP approaches to surgically drain RP abscesses.

\section{Material and Methods}

Data were collected and analyzed from 45 patients with RP abscess. Their condition was diagnosed using an abdominal CT scan performed at Inha University Hospital from January 2014 to August 2018. There were 26 patients with RP abscesses involving $2 \mathrm{RP}$ zones and systemic inflammatory response syndrome, of which there were 4 patients excluded: 2 of them refused surgery, and the other 2 could not undergo surgery due to septic shock. The study population comprised of 22 patients who received emergency surgery, due to their septic condition. Surgical drainage was performed using either the TP or RP approach method.

The positioning of the patient for the RP approach was the supine position. An incision was made from the posterior half of the 12th rib to the lateral border of the rectus abdominis muscle. The internal and external oblique and transversus abdominus muscles were divided. The peritoneum was mobilized medially to gain exposure to the abscess pocket. The positioning of the patient for the TP approach was also the supine position. A midline skin incision or paramedian incision near the abscess pocket was made. The internal organ was mobilized medially or laterally to gain exposure of the abscess pocket. Subsequently, the abscess was approached on the lateral side of the paracolic gutter.

The characteristics, etiology, laboratory and radiological findings, surgical techniques, surgical complications, and total number of hospital days, including those from other hospitalizations were examined. The patients were assigned to either the TP or RP approach group and mortality, complications, and number of hospital days were compared between the 2 groups.
The primary endpoint was the determination of the more effective procedure for patients with RP abscess. The secondary endpoint was to compare the mortality rate, reintervention rate, and complications of the surgical drainage of RP abscess between the TP and RP approaches.

Statistical analysis was conducted using MedCalc Statistical Software Version 19.2.1 (MedCalc Software Ltd, Ostend, Belgium; https://www.medcalc.org; 2020). Categorical variables were represented as mean values with ranges, and qualitative variables were described as a frequency and percentage. The Fisher's exact test and Student $t$ test were used to assess the differences in reintervention rate, mortality rate, and hospital length of stay. Values for $p$ less than 0.05 were considered statistically significant.

\section{Results}

There were 22 patients treated with surgical drainage who were assigned to a group depending on the type of surgical approach, that is, TP or RP. The TP and RP approach groups had 13 and 9 patients, respectively. The characteristics of patients (age, sex, etiology, pathogen, and comorbidity) from the 2 groups were not different. There was no significant difference in the mean age. The mean ages of the TP and RP groups were 72 and $75(p=0.864)$, respectively. Furthermore, there was no significant difference in distribution of sex between the 2 groups (males: 9 vs. $6 ; p=0.618$ ).

The etiology of RP abscess included musculoskeletal, urological, hepatobiliary, intestinal, and anorectal problems. There were no differences in etiology between the 2 groups $(p=0.754)$. After drainage of the RP abscess, the causal pathogen was confirmed. The total number of gram-negative bacilli (GNB) pathogens was 11: 6 in the TP group and 5 in the RP. On the other hand, gram-positive cocci (GPC) pathogens were also confirmed in 5 patients: 3 from the TP group and 2 from the RP group ( $p=0.475)$.

The period from surgery to death was as follows: $18,22,33$, 37, and 62 days for 5 patients from the TP group, and 29 days

Table 1. A comparison of the outcomes for patients who underwent retroperitoneal abscess drainage using the transperitoneal approach or the retroperitoneal approach.

\begin{tabular}{lccc} 
& $\begin{array}{c}\text { Transperitoneal approach } \\
(n=13)\end{array}$ & $\begin{array}{c}\text { Retroperitoneal approach } \\
(n=9)\end{array}$ & $p$ \\
\hline Reintervention & $4(30.7)$ & $6(66.6)$ & 0.02 \\
Mortality & $5(38.4)$ & $1(11.1)$ & 0.01 \\
Hospitalized days (range) & $58(18-280)$ & $76(29-180)$ & 0.03 \\
\hline
\end{tabular}

Data are presented as mean or $n$ (\% or range).

$p \leq 0.05$ were considered to be statistically significant. 
for 1 patient from the RP group. Causes of death in 3 patients from the TP group were progression of sepsis due to peritonitis, and 2 who died of heart failure. The cause of death in the RP group patient was colon necrosis due to postoperative bowel ischemia.

Comorbidity included several factors, such as diabetes, malignancy, chronic renal failure, chronic liver disease, and immunosuppression. However, there was no difference in these factors between the 2 groups ( $p=0.814$ ). However, a significant difference was observed in the mortality rate between the 2 groups ( $p<0.01$; Table 1 ). The reintervention number in the RP group $(n=6)$ was significantly higher than in the TP group $(n=4$, $p=0.02$; Table 1). The total mean number of hospital days of the RP group (mean: 76, range: 29-180 days) were more than the TP group (mean: 58 , range: $18-280$ days, $p=0.03$; Table 1 ).

\section{Discussion}

Infection of the RP space is associated with a delay in diagnosis and a high mortality rate, varying from $22 \%$ to $46 \%$ [4]. Typical symptoms include nonspecific, nonlocalized abdominal pain, variable gastrointestinal complaints, and generalized symptoms, such as chills, sweating, fever, or malaise. Approximately $10 \%$ of patients are asymptomatic [5]. Due to the various, nonspecific symptoms or asymptomatic status associated with RP abscess, it can be misdiagnosed. Therefore, the gradual onset and late diagnosis of RP abscesses can lead to inappropriate treatment. For example, patients with diabetes mellitus have a high risk of infection, and it is very difficult to diagnose considering its increasing morbidity and mortality [6].

The diagnostic tools for RP abscess include laboratory and radiological examinations. However, laboratory examinations are of little value in specifying the diagnosis, and it is the use of CT which has greatly improved radiological diagnostic accuracy [7]. The CT technique is more sensitive and specific, with a diagnostic performance in the range of $90-100 \%$. The advantages of CT are that it allows the diagnosis and precise delimitation of small pockets of infection (1-2 cm) as well as the detection of septation or lobulation. Thus, CT is considered a major diagnostic tool [8].

The definitive treatment for RP abscess is surgical drainage. Some studies have shown that percutaneous drainage is a feasible and safe treatment option for small abscesses or in patients who cannot undergo surgery due to poor medical conditions $[9,10]$. However, from these studies on RP abscess, comparisons of the outcomes of surgical methods was limited. Therefore, the current study compared the mortality, complications, and number of hospital days between the TP and RP approach groups.

The source of infection may be primary or secondary [11]. A primary infection is hematogenously disseminated and usually located in the muscle or spine. Traditionally, a RP abscess is associated with a staphylococcal infection and is caused by hematogenous dissemination from a skin focus [12]. Contrastingly, a secondary infection involves infection from organs within the RP space. For example, pyelonephritis, secondary to a genitourinary infection was reported to

Table 2. Patient demographics.

\begin{tabular}{|c|c|c|c|}
\hline & $\begin{array}{l}\text { Overall } \\
(n=22)\end{array}$ & $\begin{array}{l}\text { Transperitoneal approach } \\
(n=13)\end{array}$ & $\begin{array}{l}\text { Retroperitoneal approach } \\
(n=9)\end{array}$ \\
\hline Males & $15(68.1)$ & $9(69.2)$ & $6(66.6)$ \\
\hline Age (y) & $73.2(48-87)$ & $72(56-87)$ & $75(48-86)$ \\
\hline \multicolumn{4}{|l|}{ Comorbidity } \\
\hline Diabetes & $13(59.0)$ & $7(53.8)$ & $6(66.6)$ \\
\hline Malignancy & $6(27.2)$ & $4(30.7)$ & $2(22.2)$ \\
\hline CKD & $3(13.6)$ & $2(15.3)$ & $1(11.1)$ \\
\hline CLD & $4(18.1)$ & $3(23.0)$ & $1(11.1)$ \\
\hline Immunocompromised & $3(13.6)$ & $2(15.3)$ & $1(11.1)$ \\
\hline \multicolumn{4}{|l|}{ Pathogen } \\
\hline GNB & $11(50.0)$ & $6(46.1)$ & $5(55.5)$ \\
\hline GPC & $5(22.7)$ & $3(23.0)$ & $2(22.2)$ \\
\hline Other & $6(27.2)$ & $4(30.7)$ & $2(22.2)$ \\
\hline
\end{tabular}

Data are presented as mean $n$ (\% or range).

$\mathrm{CKD}=$ chronic kidney disease; $\mathrm{CLD}$ = chronic liver disease; GNB = gram-negative bacilli; GPC = gram-positive cocci. 
be the most common etiology of RP abscess, followed by gastrointestinal infection (diverticulitis, RP appendicitis, pancreatitis, biliary infection, and peptic ulcer diseases) [13]. However, GNB are the most frequent pathogens, and these abscesses are caused by disease of the kidneys, other organs, or neighboring anatomic structures: duodenum, pancreas, colon, and lumbar spine. In this study, the most frequent microorganisms isolated from the abscess material were GNB (GNB:11, GPC:5; Table 2). This result was also confirmed in other studies $[8,10]$. Of the 6 patients in the other group, mycobacteria were isolated from an abscess originating from the spine in 4 patients $[5,8]$. Fungi (Candida) were isolated from 2 HIV-infected immunocompromised patients.

There was no difference in early antibacterial treatment depending on the type of organism. Broad-spectrum antibiotics were used with surgery in the diagnosis of RP abscess. After the result of abscess material culture was confirmed, the antimicrobial agent was changed. Unlike other studies, polymicrobial infections were not detected. In other studies, it was proposed that rapid surgical exploration and treatment had contributed to preventing polymicrobial infection [9]. However, in the current study, it was difficult to know how long it took before symptoms appeared and tests and surgical intervention were performed. Therefore, it is difficult to determine why polymicrobial infection was not detected. Further research and analysis are required to confirm these results.

There was no difference in mortality and hospital stay between pathogen groups. The overall mortality rate $(27 \%)$ in this current series was higher than reported in previous studies $[8,9]$ but similar to those yielded in another study series [5]. The reason for a higher mortality rate than previously reported may be due to number of elderly patients and comorbidities in this current study.

There were 5 deaths in the TP group and 1 death in the RP group, and the statistical difference between the 2 groups, depending on the period, was not calculated. It is difficult to generalize the cause of death. In the TP group, the method of surgery itself can cause severe peritoneal inflammation. The cause of death in the RP group was organ damage caused by major vascular damage of the retroperitoneum during the operation.

The TP group was characterized by a higher mortality rate and fewer number of hospital days. It was postulated that the patients in the TP group died early, resulting in a short, average hospital stay. The reintervention rate was high in the RP group. It was hypothesized that various RP structures had become obstacles to the pus draining and that it was difficult for antibacterial agents to reach the avascular RP space. Eventually, if the drainage of pus was not good, the number of hospital days for treatment increased, and the procedure was performed again.

Surgical drainage of RP abscess is more effective than medical treatment. The patients were assigned to the TP approach or the RP approach group according to the need for surgical drainage. The results of the TP and RP approaches have valuable significance (Table 1). In conclusion, the TP approach needs more careful access than the RP approach because it has a higher mortality rate than the RP approach. However, the RP approach has more cases of reinterventions than the TP approach. It has been reported that the TP approach was associated with a $67 \%$ failure rate [5].

For treatment and prognosis of RP abscess, removal of the abscess is important. Thus, abscess drainage is needed. From the data in this study, it can be seen that the patients who underwent RP drainage needed more reinterventions than those treated with the TP drainage approach. Therefore, in these septic-condition patients, open abdominal surgery is not advisable, has a higher risk of mortality, and has greater morbidity. Hence, the RP approach is less risky in these patients. Patients treated with the TP drainage approach needed less reintervention than those treated with the RP approach. However, the TP approach had higher mortality than the RP approach in this study.

\section{Conclusion}

RP abscesses are difficult to diagnose and treat, and have a poor prognosis and a high mortality rate. Surgical drainage is an effective treatment that has 2 different approaches: the TP and RP approaches. The TP approach is effective for the removal of RP abscesses. However, the TP approach can cause multiple bowel injuries and inflammation spreads into the abdominal cavity. Patients who were in a septic condition had a higher risk of mortality due to open abdominal surgery. The $\mathrm{RP}$ approach leaves the bowel intact without injuring it, as the peritoneum is lifted along with the inflamed bowel, which acts as a pseudo-capsule around it. Therefore, intestinal injury is avoided. In addition, the spread of inflammation into the abdominal cavity can be avoided. However, this approach has a disadvantage in that it requires more reintervention events. Such a study, comparing surgical drainage methods for RP abscess, had not been conducted before. Therefore, this study is novel and reports valuable and meaningful findings regarding this procedure.

\section{Conflicts of Interest}

The authors have no conflicts of interest to declare. 


\section{References}

[1] Hamdy FC, Eardley I. Oxford textbook of urological surgery. Oxford (UK): Oxford University Press; 2017. p. 45-50.

[2] Brolin RE, Nosher JL, Leiman S, Lee WS, Greco RS. Percutaneous catheter versus open surgical drainage in the treatment of abdominal abscesses. Am Surg 1984;50(2):102-8.

[3] Alimi YS, Hartung O, Orsoni P, Juhan C. Abdominal aortic laparoscopic surgery: retroperitoneal or transperitoneal approach? Eur J Vasc Endovasc Surg 2000;19(1):21-6.

[4] Harris LF, Sparks JE. Retroperitoneal abscess. Case report and review of the literature. Dig Dis Sci 1980;25(5):392-5.

[5] Crepps JT, Welch JP, Orlando R 3rd. Management and outcome of retroperitoneal abscesses. Ann Surg 1987;205(3):276-81.

[6] Meshikhes A-WN, Al-Otaibi MM, Al-Amer HA, Al-Saif OH. Retroperitoneal abscess as an initial presentation of cecal carcinoma. Saudi Med J 2002;23(8):999-1001.
[7] Wolverson MK, Jagannadharao B, Sundaram M, Joyce PF, Riaz MA, Shields JB. CT as a primary diagnostic method in evaluating intraabdominal abscess. Am J Roentgenol 1979;133(6):1089-95.

[8] Edelstein H, McCabe RE. Perinephric abscess. Modern diagnosis and treatment in 47 cases. Medicine (Baltimore) 1988;67(2):118-31.

[9] Winter B, Gajda M, Grimm M-O. Diagnosis and treatment of retroperitoneal abscesses. Der Urologe A 2016;55(6):741-7. [in German].

[10] Fowler JE Jr, Perkins T. Presentation, diagnosis and treatment of renal abscesses: 1972-1988. J Urol 1994;151(4):847-51.

[11] Huang SW, Lo WO, Lin CM, Hsieh TS, Wang SF, Tsai SW, et al. Retroperitoneal abscess: 7-year experience of 29 cases in tertiary care center in Taiwan. Urol Sci 2015;26(3):218-21.

[12] Alcina EL, Guzman SA, Monfort MJJ, Escriva AF, Cruz FJ. Abscesos renales y perirrenales. Actas urol Esp 1999;23:135-9. [in Spanish].

[13] Manjón CC, Sánchez AT, Lara JDP, Silva VM, Betriu GC, Sánchez AR, et al. Retroperitoneal abscesses-analysis of a series of 66 cases. Scand J Urol Nephrol 2003;37(2):139-44 